\title{
Clinical impact of the type VI secretion system on virulence of Campylobacter species during infection
}

Jessica Agnetti ${ }^{1}$, Helena M. B. Seth-Smith ${ }^{1,2}$, Sebastian Ursich ${ }^{3,4}$, Josiane Reist ${ }^{1,2}$, Marek Basler ${ }^{3}$, Christian Nickel ${ }^{5}$, Stefano Bassetti ${ }^{6}$, Nicole Ritz ${ }^{7,8}$, Sarah Tschudin-Sutter ${ }^{9}$ and Adrian Egli ${ }^{1,4^{*}}$

\begin{abstract}
Background: The clinical course of Campylobacter infection varies in symptoms and severity depending on host factors, virulence of the pathogen and initiated therapy. The type VI secretion system (T6SS) has been identified as a novel virulence factor, which mediates contact-dependent injection of enzymes and toxins into competing bacteria or host cells and facilitates the colonisation of a host organism. We aimed to compare the clinical course of Campylobacter infection caused by strains with and without the T6SS and identify possible associations between this putative virulence factor and the clinical manifestations of disease.

Methods: From April 2015 to January 2017, patients with detection of Campylobacter spp. were identified at the University Hospital of Basel and the University Children's Hospital of Basel and included in this case-control study. Presence of the T6SS gene cluster was assayed by PCR targeting the hcp gene, confirmed with whole genome sequencing. Pertinent clinical data was collected by medical record review. Differences in disease- and hostcharacteristics between T6SS-positive (case) and -negative (control) were compared in a uni- and multi-variable analysis. Hospital admission, antibiotic therapy, admission to intensive care unit, development of bacteraemia and in-hospital mortality were considered as clinical endpoints.

Results: We identified 138 cases of Campylobacter jejuni infections and 18 cases of Campylobacter coli infections from a paediatric and adult population. Analyses were focused on adult patients with C. jejuni $(n=119)$ of which $16.8 \%$ were T6SS-positive. Comparisons between T6SS-positive and -negative C. jejuni isolates did not reveal significant differences regarding clinical manifestations or course of disease. All clinical endpoints showed a similar distribution in both groups. A higher score in the Charlson Comorbidity Index was associated with T6SS-positive C. jejuni isolates $(p<0.001)$ and patients were more likely to have a solid organ transplant and to be under immunosuppressive therapy.

Conclusions: Our study does not provide evidence that T6SS is associated with a more severe clinical course. Interestingly, T6SS-positive isolates are more commonly found in immunocompromised patients: an observation which merits further investigation.
\end{abstract}

Keywords: Type IV secretion system, Campylobacter jejuni, Campylobacter coli, Virulence, Diarrhoea, Infection, Clinical course

\footnotetext{
* Correspondence: adrian.egli@usb.ch

${ }^{1}$ Applied Microbiology Research, Department of Biomedicine, University of Basel, Basel, Switzerland

${ }^{4}$ Current address: Institute for Molecular Cancer Research IMCR, University of

Zürich, Zürich, Switzerland

Full list of author information is available at the end of the article
}

(c) The Author(s). 2019 Open Access This article is distributed under the terms of the Creative Commons Attribution 4.0 International License (http://creativecommons.org/licenses/by/4.0/), which permits unrestricted use, distribution, and reproduction in any medium, provided you give appropriate credit to the original author(s) and the source, provide a link to the Creative Commons license, and indicate if changes were made. The Creative Commons Public Domain Dedication waiver (http://creativecommons.org/publicdomain/zero/1.0/) applies to the data made available in this article, unless otherwise stated. 


\section{Background}

Infection with Campylobacter spp. is a worldwide leading cause of diarrhoea, showing an increase in incidence over the last decade in North America, Europe and Australia [1]. In Switzerland, as in other European countries, campylobacteriosis is currently the most commonly reported food-borne bacterial disease [2] with up to $70 \%$ of all cases attributable to chicken consumption [3], thus posing a relevant public health problem. In Switzerland, the incidence in 2017 was 85.4 cases per 100,000 people [4] and the annual economic burden due to laboratory-confirmed campylobacteriosis has recently been estimated at around $€ 8.3$ million [5]. It is assumed that a large number of additional cases go unreported [5].

The clinical course of campylobacteriosis varies in symptoms and severity. Infection with Campylobacter jejuni - the most frequently diagnosed species - is most often characterised by acute diarrhoea, fever, and abdominal pain [6]. In most cases self-limiting, symptoms typically last from one day to a week, however, may persist for longer in $10-20 \%$ of patients and relapse may occur in another 5-10\% [6]. The disease is occasionally further complicated by bacteraemia and sepsis or by extra-gastrointestinal manifestations following enteric disease, such as reactive arthritis and Guillain-Barré syndrome [6]. Complications and prolonged disease usually affect elderly or immunocompromised patients with comorbidities [1].

The clinical manifestation of infection with Campylobacter spp. is determined by host factors such as immune-competency, age, genetic background, and bacterial factors such as virulence of the pathogen, initial infectious dose, and initiated therapy [6, 7]. Current research has identified novel mechanisms that may contribute to virulence and survival of Campylobacter spp. in humans, such as the Type VI Secretion System (T6SS) [8], one of several bacterial systems for protein secretion [9]. With a structure similar to the tail of the contractile bacteriophages [10], the T6SS allows the contact-dependent secretion of effectors into other bacterial or host cells [11, 12]. The T6SS is encoded by a gene cluster of approx.17kb comprising 13 genes [13]. One of the hallmark genes of the T6SS gene cluster is $h c p$ (encoding the secreted component hemolysin co-regulated protein) [14], which has been defined as a marker of a complete T6SS [15].

The expression of T6SS in C. jejuni has been demonstrated to be of importance for host cell adhesion and invasion in vitro: mutants with a defective secretion system show an up to $50 \%$ reduced ability to adhere to and invade colonic epithelial cells and macrophages [13]. Furthermore, in vivo studies show a higher persistence of $C$. jejuni expressing T6SS, with the dynamics of T6SS-regulation favouring colonisation of the colon $[12,13]$. In polymicrobial environments such as the gastrointestinal tract, the T6SS also presents an advantage by allowing the pathogen to overcome the local microbiota [16].
In view of the diverse functionality of T6SS and extensive in vitro/in vivo research, the secretion system may provide a survival benefit for Campylobacter spp. contributing to successful and persistent infection [17]. A possible connection between T6SS as a clinically relevant virulence factor in Campylobacter spp. and manifestation of disease in humans has been insufficiently studied so far. Harrison et al. [15] found a correlation between T6SS-positive C. jejuni strains and bloody diarrhoea, and accordingly more severe disease, in a sample of 36 patients from Vietnam. A causal relationship could, however, not be proven [15].

In this study, we aimed to compare the clinical course of Campylobacter infection caused by strains with and without T6SS in an observational case-control study, and to identify possible associations between this virulence factor and the clinical manifestations of disease.

\section{Methods}

Cases with detection of Campylobacter in any clinical isolate at the University Hospital Basel within the time period from April 2015 to January 2017 were eligible for this observational case-control study. A total of 119 consecutive C. jejuni and 15 C. coli bacteria were isolated. Coinfections were excluded. Rare Campylobacter species such as $C$. fetus $(n=2), C$. upsaliensis $(\mathrm{n}=2)$, and $C$. concisus $(n=1)$ were also detected, but subsequently excluded from the analysis due to the small sample size. Additionally, 22 Campylobacter isolates were detected at the University Children's Hospital Basel during the same observation period. Due to small sample size, these data were only analysed descriptively and not included in the case-control study.

\section{Patients and data collection}

The study has been approved by the local ethical review board (EKNZ, approval number 2016-01183). The medical records of the included patients were retrospectively reviewed. Data extraction was performed blinded for T6SS status. Hospitalised cases included in this study were admitted via the emergency unit. Outpatients were most commonly treated in the emergency unit and in some cases monitored for a maximum of one night.

The following data were systematically collected: demographic characteristics, medical and travel history as pertaining to the present infection. In addition, clinical characteristics at presentation, characteristics of diarrhoeal disease, and laboratory results at presentation, as well as the requirement for antibiotic treatment, were documented. Underlying diseases were recorded according to the Charlson Comorbidity Index [18-21]. Regarding treatment, fluid substitution and antibiotic therapy (including duration and application) were recorded. Information on the need for and duration of hospitalisation, need for ICU-stay and in-hospital mortality were extracted. Microbiological data included identification to 
the species level and the antibiotic susceptibilities to erythromycin and ciprofloxacin $(\mu \mathrm{g} / \mathrm{mL})$.

The following categorical factors were defined as primary clinical endpoints: need for hospital admission, antibiotic therapy, intensive care unit (ICU) stay, development of bacteraemia, and in-hospital mortality.

\section{Isolate growth and identification}

All routine clinical microbiology was performed in an ISO/IEC 17025 accredited laboratory. Stool samples were cultured in microaerobic conditions (5\% O2, $10 \%$ $\mathrm{CO} 2$ and $85 \%$ N2) on Campylosel agar plates (bioMérieux, Marci I'Étoile, France) at $41{ }^{\circ} \mathrm{C}$ for $48 \mathrm{~h}$. Bacterial isolates were identified using Matrix Assisted Laser Desorption Ionization - Time of Flight mass spectrometry using methods described by Bessède et al. [22] on a Microflex LT mass spectrometer and MALDI Biotyper Compass software v. 4.1.70 (Bruker Daltonics, Germany). The value of "Score" $\geq 2.0$ was accepted as a measure for reliable species identification.

\section{Minimal inhibitory concentration (MIC) determination MICs for erythromycin and ciprofloxacin of each isolate were determined by E-test (bioMérieux) and incubated under microaerobic conditions on MHF (Müller Hinton agar $+5 \%$ horse blood $+20 \mathrm{mg} / \mathrm{l} \beta$-NAD) agar plates (bioMérieux) at $41{ }^{\circ} \mathrm{C}$ for $24 \mathrm{~h}$. The interpretation of MICs was performed according to EUCAST guidelines (version 8.1; www.eucast.org).}

\section{Type VI secretion system (T6SS) PCR detection}

PCR detection of the $h c p$ gene as a locus representing the T6SS in C. jejuni and C. coli isolates was performed [23]. We used C. jejuni subsp. jejuni strains from ATCC (Ref. 33560 and 43431) as positive controls. Briefly, the DNA of cultured isolates was extracted using the DNA Bacterial card on the EZ1 Advanced XL automated extraction system (Qiagen, Hombrechtikon) using the manufacturer's protocol following a digestion step using proteinase $\mathrm{K}$ performed for $10 \mathrm{~min}$ at $56^{\circ} \mathrm{C}$ and $10 \mathrm{~min}$ at $95^{\circ} \mathrm{C}$. The PCR used the following primers: Hcp_F $5^{\prime}$-CAAGCGGTG CATCTACTGAA-3', Hcp_R 5'-TAAGCTTTGCCCTC TCTCCA-3' and as a control the gltA gene gltA_F 5'-GCCCAAAGCCCATCAAGCGGA-3', gltA_R 5'-GC GCTTTGGGGTCATGCACA-3'. HotStarTaq (Qiagen) was used for the PCR with a melting temperature of $57^{\circ} \mathrm{C}$. PCR fragments ( $h c p 463 \mathrm{bp}$ and $g l t A 142 \mathrm{bp}$ ) were detected on a 2200 TapeStation instrument (Agilent, Santa Clara, USA) using the D1000 ScreenTape assay (Agilent).

\section{Whole genome sequencing (WGS)}

Isolate details are given in Additional file 1: Table S1. DNA was extracted as above. Library generation with Nextera XT (Illumina, Cambridge, UK) was followed by sequencing $2 \times 300$ bp on an Illumina Miseq platform. Downstream analysis was performed in CLC Genomics Workbench v 10.1.1 with read trimming and QC, followed by coverage determination by mapping against the genome of NCTC11168 (accession number NC002163). All genomes gave a mean coverage over $62 x$, up to $488 x$. The presence of the T6SS was determined by mapping against the well characterised T6SS locus in strain 414 (accession number CM000855, locus tags C414_000040086C414_000040100) [13]. All isolates with reads mapping to under $25 \%$ of the gene cluster, also with mean coverage of the region lower than $1 \mathrm{x}$, were considered as negative ( $n$ $=103$ ); those mapping to over $86 \%$ of the locus were considered to be positive $(n=21)$; and those mapping to $26-$ $41 \%$ of the locus considered as partial $(n=10)$. Read data generated for this study can be found in the European $\mathrm{Nu}$ cleotide Archive (ENA, http://www.ebi.ac.uk/ena/) under project number PRJEB30092.

\section{Statistical methods}

Descriptive analysis was performed with all data. For the analysis of the clinical impact of T6SS, only adult patients infected with $C$. jejuni were included into the statistical model to obtain a more homogenous cohort. Median and interquartile ranges are shown, unless otherwise indicated. Disease and host characteristics were compared in a univariate analysis between T6SS-positive (case) and negative strains (controls). For the calculation of adjusted odds ratios, a multivariable logistic regression model was constructed using variables that were statistically significantly associated with a positive T6SS status in the univariate analysis. Results with $p$-values $<0.05$ (two-sided) were considered statistically significant.

\section{Results}

\section{C. jejuni isolates are more often positive for T6SS than $C$. coli}

From April 2015 to January 2017, we identified a total of 119 cases of $C$. jejuni infection and 15 cases of C. coli infection from an adult population. All cases were community acquired and no nosocomial infections occurred. Six of 119 C. jejuni strains (5.0\%) were isolated from blood culture. In two patients with bacteraemia, stool culture was also positive for the same species.

All isolates from the adult population $(n=134)$ were tested for the presence of the T6SS, first by PCR targeting the $h c p$ gene, and also by WGS. Data agreed in all cases. Overall, 21 isolates $(15.7 \%)$ were found to be T6SS positive. Regarding species, 20/119 (16.8\%) C. jejuni and 1/15 (6.7\%) C. coli isolates were found to be T6SS positive. In 10 cases, partial mapping to the T6SS was observed, with reads mapping to the locus tags C414_000040089 and C414_000040100, but not to $h c p$ (C414_000040085). Interestingly, mapping coverage to the T6SS in the positive isolates represented a relative 
coverage of 1-36\% compared to mapping across the whole genome (Additional file 1: Table S1).

Additionally, in a paediatric population, 19/22 (86.4\%) C. jejuni and 3/22 (13.6\%) C. coli were isolated. The isolates were screened by PCR for the hcp gene locus, which was found in 3/19 (15.8\%) C. jejuni strains. Due to the low frequencies of isolates from paediatric patients and the obvious expected differences in both cohorts, we focused our further analysis on bacterial isolates from adult patients. Additional file 2: Table S2 provides the clinical characteristics of children infected with Campylobacter spp.

\section{T6SS positive $C$. jejuni isolates are associated with resistance towards ciprofloxacin}

All Campylobacter strains from the adult population were tested for erythromycin and ciprofloxacin susceptibility. Two of 119 C. jejuni (1.7\%) and three of 15 C. coli (20.0\%) had resistant MICs for erythromycin. 72 of 119 C. jejuni (60.5\%) and 10 of 15 C. coli (66.7\%) had resistant MICs for ciprofloxacin. All 20 T6SS-positive $C$. jejuni strains were susceptible to erythromycin, whereas 17 of 20 T6SS-positive C. jejuni strains (85.0\%) were resistant to ciprofloxacin (Chi-squared, $p=0.01$ ).

\section{Clinical data}

Clinical characteristics of infection in adult patients with $C$. coli and C. jejuni are listed in Table 1. Infection with C. jejuni manifested in 117/119 cases, and in all 15 C. coli cases, as gastrointestinal disease with diarrhoea, often accompanied by abdominal pain ( 68.1 and $46.7 \%$, respectively) and vomiting (28.6 and $20.0 \%$, respectively). Patients also commonly reported fever (52.1 and 46.7\%, respectively).

In patients with $C$. jejuni infection, underlying diseases were present in $47.9 \%$. Malignancies (including solid neoplasia, leukaemia and lymphoma) and diabetes mellitus type 2 were identified as the leading comorbidities. Eleven patients had an immunocompromised status due to a solid organ transplant, allogenic haematologic stem cell transplant (HSCT) or AIDS. The course of disease was uncomplicated in most cases. $61.3 \%$ of patients had to be hospitalised. Two patients were admitted to ICU, one bacteraemic patient died at the hospital. Table 2 compares hospitalised patients with patients receiving outpatient care. Hospitalised patients were older, showed a higher leucocyte count and higher CRP-values, which are all indicators of a more severely affected cohort. Creatinine clearance was lower in hospitalised patients.

\section{Comparisons between patients infected with $C$. jejuni harbouring and not harbouring T6SS}

Due to the low frequency of T6SS-positive C. coli strains, only $C$. jejuni were included in the final statistical model. We analysed the $C$. jejuni adult cohort $(n=119)$ with respect to the T6SS status of each isolate, to ascertain possible associations with the virulence factor. Table 3 shows a comparison of patients infected with T6SS-positive versus T6SS-negative $C$. jejuni isolates, focusing on patient characteristics and clinical course of disease. None of the defined clinical endpoints was associated either with T6SS-positive or negative C. jejuni isolates: $60.0 \%$ of patients with T6SS-positive isolates and $61.6 \%$ with T6SS-negative isolates were admitted to the hospital, respectively. Antibiotic therapy was administered to $65.0 \%$ of patients infected with T6SS-positive and in $60.6 \%$ of patients with T6SS-negative isolates. One of 20 patients with a T6SS-positive isolate and one of 99 patients with a T6SS-negative strain was admitted to ICU. Of note, bacteraemia was present only in patients infected with T6SS-negative strains $(n=6)$, leading to a single patient death in hospital.

Patients infected with T6SS-positive C. jejuni isolates had a higher score in the Charlson Comorbidity Index, were more likely to have had a solid organ transplant, or to be under immunosuppressive therapy as compared to patients infected with T6SS-negative isolates. A higher comorbidity score remained associated with infection with a T6SS-positive strain in multivariable analysis including

Table 1 Adult patient characteristics

\begin{tabular}{llll}
\hline Characteristics & $\begin{array}{l}\text { Patients with C. coli infection } \\
(n=15)\end{array}$ & $\begin{array}{l}\text { Patients with C. jejuni infection } \\
(\mathrm{n}=119)\end{array}$ & $\begin{array}{l}\text { All adult patients } \\
(\mathrm{n}=134)\end{array}$ \\
\hline Age (median years) & $51(26-80)$ & $57(33-74)$ & $55(33-74)$ \\
Male gender & $7(46.7)$ & $74(62.2)$ & $81(60.4)$ \\
Hospitalised patients & $7(46.7)$ & $73(61.3)$ & $80(59.7)$ \\
Duration of hospitalisation (median days) & $0(0-6)$ & $1(0-5)$ & $1(0-5)$ \\
Fever history (yes) & $7(46.7)$ & $62(52.1)$ & $69(51.5)$ \\
Bloody diarrhoea (yes) & $2(13.3)$ & $21(17.6)$ & $23(17.2)$ \\
Antibiotic therapy (yes) & $9(60.0)$ & $73(61.3)$ & $82(61.2)$ \\
Leucocytes $(\times 10 \wedge 9 /$ /) & $8.5(5.0-12.1)$ & $8.6(5.9-11.2)$ & $8.5(5.9-11.3)$ \\
C-reactive protein $(\mathrm{mg} / \mathrm{l})$ & $69(41-186)$ & $66(29-129)$ & $68(31-133)$ \\
\hline
\end{tabular}

Data are median (IQR) or $\mathrm{n}(\%)$ 
Table 2 Comparisons between adult patients hospitalised and receiving outpatient care with C. jejuni

\begin{tabular}{llll}
\hline & $\begin{array}{l}\text { Need for hospital admission } \\
(n=73)\end{array}$ & $\begin{array}{l}\text { Out-patient treatment } \\
(n=46)\end{array}$ \\
\hline Male gender & $48(65.8)$ & $26(56.5)$ & $45(27-63)$ \\
Age (median years) & $69(49-81)$ & $75(5.6-10.2)$ & 0.001 \\
Leucocytes $(\times 10 \wedge 9 / \mathrm{l})$ & $9.2(6.7-11.9)$ & $43(10-87)$ & 0.05 \\
C-reactive protein $(\mathrm{mg} / \mathrm{l})$ & $84(39-171)$ & $88(71-108)$ & 0.001 \\
Creatinine clearance $(\mathrm{ml} / \mathrm{min})$ & $68(41-95)$ & & 0.004 \\
\hline
\end{tabular}

Data are median (IQR) or $\mathrm{n}(\%) . P$ values $\leq 0.05$ are shown

the CCI, solid organ transplants and immunosuppressive therapy in the final regression model (Table 4).

\section{Discussion}

This study adds to the growing amount of research on the bacterial T6SS providing insight into its occurrence in clinical Campylobacter spp. and examining its role in human infection.

While $15.7 \%$ of the isolates from the adult population were positive for the complete T6SS locus, a proportion also carry partial T6SS gene clusters, as has previously been noted [15, 24]. These were not identified by PCR primers targeting $h c p$, meaning that the PCR of $h c p$ is a valuable marker for a functional T6SS [15]. That mean coverage of the T6SS is lower than that across the genome may be a sequencing artefact relating to the relatively low $\% \mathrm{G}+\mathrm{C}$ content of this locus, or may indicate that the locus is lost from some bacteria during culture.
The clinical characteristics of infection with Campylobacter spp. in the study population are similar to those previously described by various authors $[1,6]$. Infection with $C$. jejuni and $C$. coli were clinically indistinguishable: both manifested as acute gastroenteritis with a similar distribution in both species causing vomiting, abdominal pain, bloody diarrhoea and fever history. Complications arose in very few cases, with a single death attributable to invasive campylobacteriosis. In view of epidemiologic studies reporting a peak incidence of Campylobacter infection in toddlers and young adults $[1,25,26]$, the median age of 55 in patients in the present study seems relatively high. However, Schmutz et al. [27] describe a trend towards a higher median age in Campylobacter infections in Switzerland. A possible selection bias towards an older and sicker population remains: $59.7 \%$ of all diagnosed patients required hospital admission and antibiotic therapy was prescribed in $61.2 \%$ of cases. Furthermore, $5.0 \%$ of patients infected with

Table 3 Comparisons between adult patients infected with T6SS-positive and T6SS-negative C. jejuni

\begin{tabular}{|c|c|c|c|}
\hline & T6SS-positive & T6SS-negative & $p$-value \\
\hline & $(n=20)$ & $(n=99)$ & \\
\hline \multicolumn{4}{|l|}{ Baseline characteristics } \\
\hline Age (median years) & $58(46-77)$ & $57(31-74)$ & n.s. \\
\hline Male gender & $13(65.0)$ & $61(61.6)$ & n.s. \\
\hline Hospitalised patients & $12(60.0)$ & $61(61.6)$ & n.s. \\
\hline Duration of hospital stay (median days) & $3(0-5)$ & $1(0-5)$ & n.s. \\
\hline ICU stay (yes) & $1(5.0)$ & $1(1.0)$ & n.s. \\
\hline \multicolumn{4}{|l|}{ Comorbidities } \\
\hline Charlson Comorbidity Index & $2(1-5)$ & $0(0-1)$ & $<0.001$ \\
\hline Solid organ transplant & $4(20.0)$ & $3(3.0)$ & 0.015 \\
\hline Immunosuppressant therapy & $6(30.0)$ & $9(9.1)$ & 0.019 \\
\hline \multicolumn{4}{|l|}{ Clinical characteristics } \\
\hline Bacteraemia & $0(0.0)$ & $6(6.1)$ & n.s. \\
\hline Fever history (yes) & $6(30.0)$ & $56(56.6)$ & 0.048 \\
\hline Bloody diarrhoea (yes) & $2(10.0)$ & $19(19.2)$ & n.s. \\
\hline Duration of diarrhoea (days) & $6(4-10)$ & $6(4-9)$ & n.s. \\
\hline Leucocytes $\left(\times 10^{9} / 1\right)$ & $8.5(6.1-12.1)$ & $8.7(5.9-11.1)$ & n.s. \\
\hline Antibiotic therapy (yes) & $13(65.0)$ & $60(60.6)$ & n.s. \\
\hline
\end{tabular}

Data are median (IQR) or $\mathrm{n}(\%) . P$ values $\leq 0.05$ are shown. Only $p$-values of significant results were shown. $n$.s. non-significant 
Table 4 Uni- and multivariable logistic regression models

\begin{tabular}{|c|c|c|c|c|c|c|}
\hline & \multicolumn{3}{|c|}{ Crude } & \multicolumn{3}{|c|}{ Adjusted $^{a}$} \\
\hline & OR & $95 \% \mathrm{Cl}$ & $p$-value & OR & $95 \% \mathrm{Cl}$ & $p$-value \\
\hline Charlson Comorbidity Index & 1.71 & $1.30-2.25$ & $<0.001$ & 1.65 & $1.25-2.18$ & $<0.001$ \\
\hline Solid organ transplant & 8.08 & $1.65-39.54$ & 0.010 & 1.82 & $0.19-17.83$ & 0.608 \\
\hline Immunosuppression & 4.33 & $1.34-14.05$ & 0.015 & 2.41 & $0.41-14.10$ & 0.328 \\
\hline Antacids & 2.6 & $0.98-6.89$ & 0.055 & & & \\
\hline
\end{tabular}

${ }^{a}$ Charlson Comorbidity Index, solid organ transplant and immunosuppressants were included in the final regression model

OR odds ratio, $95 \% \mathrm{Cl} 95 \%$ confidence interval

C. jejuni had a positive blood culture, which is unusually high considering population-based studies from Denmark [28] and the USA [29]. These have described an incidence of Campylobacter bacteraemia of less than $1 \%$ of all infections. Besides the aforementioned selection bias, this discrepancy may also reflect the higher number of automatic blood cultures performed in a hospital setting.

Various studies have investigated the epidemiology of T6SS in C. jejuni. The T6SS seems to be more prevalent in isolates from Asia and Egypt than Europe, with 60.6\% of human isolates T6SS-positive in Vietnam [15], 33.3\% in Thailand [15] and 57.6\% in a large Egyptian paediatric cohort [30]. European studies have shown a prevalence of $2.6 \%$ in human isolates from the UK [15] and $14 \%$ in chicken isolates from Spain [24]. The results of the present study are in accordance with these studies in that they uphold the large difference between Asian and European strains. With a T6SS-prevalence of $16.8 \%$ in $C$. jejuni the results are close to those from Spain [24], although their strains were obtained from poultry and sewerage water. Bleumink-Pluym et al. also identified a T6SS-prevalence of about $10 \%$ in strains from various sources such as human, chicken, swine, and cattle [14].

The reported prevalence of T6SS in $C$. coli reaches from $18.0 \%$ in isolates from a paediatric cohort [30] to $56.1 \%$ in isolates from retail chicken [23]. Corcionivoschi et al. [23] found a much higher percentage of T6SS-positive C. coli in comparison with C. jejuni. As they point out, only a small percentage of Campylobacter infections in humans are caused by $C$. coli, whereas the prevalence of $C$. coli and $C$. jejuni is almost equal in their study [23]. The present study identified only one functional T6SS among $15 \mathrm{C}$. coli isolates. The low prevalence of $6.7 \%$ may be due to small sample size, however: Sainato et al. [30] also recorded a relatively low frequency of T6SS in C. coli (18\%) in comparison to $C$. jejuni $(57.6 \%)$ in clinical isolates.

Our comparison between patients with T6SS-positive and -negative $C$. jejuni isolates did not reveal a significant difference in the clinical manifestations and the course of disease. All variables defined as clinical endpoints (e.g. hospital admission, antibiotic therapy) showed a similar distribution in both groups. These findings agree with a recently published study examining 176 cases of infection with C. jejuni and 126 cases of infection with $C$. coli in a paediatric cohort from Egypt concerning the presence of the hcp gene [30]. The clinical symptoms did not differ in severity between T6SS-positive and -negative Campylobacter strains [30]. This stands in contrast to the findings of Harrison et al. [15] of a positive association of T6SS-presence in $C$. jejuni with bloody diarrhoea. Bleumink-Pluym et al. [14] noted that $50 \%$ of their T6SS-positive strains were isolated from blood, also suggesting an association with more severe disease. The studies were performed with 36 and 8 C. jejuni strains, respectively, making it possible that the dissimilarity in findings is due to small sample size and potentially other virulence factors.

We found a surprisingly stable association $(p<0.001)$ with T6SS-positive strains for a higher score in the Charlson Comorbidity Index, ascertained also in a multivariable model. Cancerous diseases and diabetes mellitus type 2 were identified as the leading comorbidities. These and other diseases assessed by the CCI show an immunosuppressing effect in patients. It seems that patients with a higher morbidity, but also with an immunocompromised status are more susceptible to $C$. jejuni strains harbouring a T6SS. The association of T6SS-positive strains with allogenic haematopoietic stem cell transplantation (HSCT), solid organ transplant and immunosuppressant therapy (though not independently) supports this hypothesis. In a study of Acinetobacter baummanii isolates, Kim et al. [31] also found an association between infection with T6SS-positive strains and HSCT and immunosuppressant therapy. Our results suggest a possible contribution of the T6SS to infection of an immunocompromised and sickly host. The mechanism, however, is not clear and should be further investigated. The identified association may, however, be influenced by selection bias towards an older and more sickly population.

The rates of antibiotic resistance identified in the present study are similar to those reported by other authors $[6,32]$ with macrolide resistance higher in $C$. coli (20.0\%) than in C. jejuni (1.7\%) and generally high resistance rates for ciprofloxacin: $61.2 \%$ of all Campylobacter 
isolates. Analysis of T6SS-positive C. jejuni strains showed a significantly higher resistance rate for ciprofloxacin. A possible explanation for this association may be found in a correlation with clonal complexes analysed by multi-locus sequencing typing. Kittl et al. [3], Collado et al. [33] and Kovac et al. [34] examined the relationship between ciprofloxacin-resistance and sequence types in Campylobacter jejuni showing a significant association with clonal complexes ST-21 [3, 33, 34], ST-353 [33, 34], ST-48 [33] and ST-464 [3]. Kittl et al. noted that the correlation was independent from the host, suggesting that certain STs may develop more easily quinolone resistance [3]. Harrison et al. [15] found a correlation between the T6SS and clonal complex ST-353. It seems that clonal complexes showing higher resistance for ciprofloxacin may also be those that are associated with carrying the T6SS.

Our study has some important limitations: the overall sample size is relatively small and for the statistical analysis we have only focused on $C$. jejuni isolates from adult patients $(n=119)$. Also, a relative high proportion of patients was hospitalised, which could reflect a more severely ill and immunosuppressed patient population at a tertiary hospital setting. Finally, we found only $20 \mathrm{C}$. jejuni isolates with a T6SS locus.

\section{Conclusions}

Overall, our study does not provide evidence that the T6SS is associated with a more severe clinical course in Campylobacter jejuni. Interestingly, T6SS positive isolates are more commonly found in immunocompromised patients: an observation which merits further investigation.

\section{Additional files}

Additional file 1: Table S1. Isolates and genome data. Description of data: List of analysed Campylobacter isolates with WGS and PCR results on the presence of T6SS. (XLSX $21 \mathrm{~kb}$ )

Additional file 2: Table S2. Paediatric patient characteristics. Description of data: Table with descriptive statistics on a paediatric cohort of 22 patients. (DOCX $13 \mathrm{~kb}$ )

\section{Abbreviations}

CCl: Charlson Comorbidity Index; EKNZ: Ethikkommission Nordwest- und Zentralschweiz; hcp: Haemolysin coregulated protein; HSCT: Haematologic stem cell transplant; ICU: Intensive care unit; PCR: Polymerase chain reaction; T6SS: Type VI secretion system; WGS: Whole genome sequencing

\section{Acknowledgements}

We thank Magdalena Schneider, Christine Kiessling, Elisabeth Schultheiss, Clarisse Straub, and Rosa-Maria Vesco (all University Hospital Basel) for excellent technical assistance.

\section{Funding}

A.E. received a salary research grant of the Swiss National Science Foundation (Ambizione PZ00P3_154709/1). There has been no impact of the funding body on design, collection, analysis and interpretation and writing of the manuscript.

\section{Availability of data and materials}

All data of the study has been included into the manuscript. Read data generated for this study can be found in the European Nucleotide Archive (ENA, http://www.ebi.ac.uk/ena/) under project number PRJEB30092.

\section{Authors' contributions}

JA collected clinical data and wrote the manuscript; HMBSS performed WGS analysis and wrote the manuscript; MB and SU provided technical advice on the hcp-PCR and reviewed the manuscript; JR collected and analysed isolates and wrote the manuscript; CN, SB, NR provided clinical inputs and reviewed the manuscript; STS performed statistical analysis; AE designed the study, provided samples and analysis and wrote the manuscript. All authors have read and approved the manuscript.

\section{Ethics approval and consent to participate}

The study has been approved by the local ethical review board (EKNZ, approval number 2016-01183). This was a retrospective study so the consent to participate was waivered by the local ethical review board. General consent was signed by a majority.

\section{Consent for publication}

Not applicable.

\section{Competing interests}

The authors declare that they have no competing interests.

\section{Publisher's Note}

Springer Nature remains neutral with regard to jurisdictional claims in published maps and institutional affiliations.

\section{Author details}

${ }^{1}$ Applied Microbiology Research, Department of Biomedicine, University of Basel, Basel, Switzerland. ${ }^{2}$ Clinical Microbiology, University Hospital Basel, Petersgraben 4, 4031 Basel, Switzerland. ${ }^{3}$ Biozentrum, University of Basel, Basel, Switzerland. ${ }^{4}$ Current address: Institute for Molecular Cancer Research IMCR, University of Zürich, Zürich, Switzerland. ${ }^{5}$ Emergency Department, University Hospital Basel, Basel, Switzerland. ${ }^{6}$ Department of Internal Medicine, University Hospital Basel, Basel, Switzerland. ${ }^{7}$ Paediatric Infectious Diseases and Vaccinology, University Children Hospital Basel, Basel, Switzerland. ${ }^{8}$ Department of Pediatrics, Royal Children's Hospital Melbourne, University of Melbourne, Parkville, Australia. Infectious Diseases and Hospital Epidemiology, University Hospital Basel, Basel, Switzerland.

Received: 30 May 2018 Accepted: 28 February 2019

Published online: 07 March 2019

References

1. Kaakoush NO, Castaño-Rodríguez N, Mitchell HM, Man SM. Global epidemiology of Campylobacter infection. Clin Microbiol Rev. 2015;28(3): $687-720$

2. Baumgartner A, Felleisen R, Gut C. Campylobacter in Switzerland — risk factors and measures for dealing with the problem. Governmental report. Bern, Switzerland: Federal Office of. Public Health. 2012

3. Kittl S, Heckel G, Korczak BM, Kuhnert P. Source attribution of human Campylobacter isolates by MLST and fla-typing and association of genotypes with quinolone resistance. PLoS One. 2013;8(11):e81796.

4. European Food Safety Authority (EFSA) and European Centre for Disease Prevention and Control (ECDC). The European Union summary report on trends and sources of zoonoses, zoonotic agents and food-borne outbreaks in 2017. EFSA J. 2018;16(12):e05500.

5. Schmutz C, Mäusezahl D, Bless PJ, Hatz C, Schwenkglenks M, Urbinello D. Estimating healthcare costs of acute gastroenteritis and human campylobacteriosis in Switzerland. Epidemiol Infect. 2017;145(4):627-41.

6. Mishu Allos B, lovine NM, Blaser MJ. Campylobacter jejuni and Related Species. In: Mandell GL, Bennett JE, Dolin R, editors. Mandell, Douglas, and Bennett's Principles and Practice of Infectious Diseases. Philadelphia, Pennsylvania: Churchill Livingstone Elsevier; 2010. p. 2485-93.

7. Janssen R, Krogfelt KA, Cawthraw SA, van Pelt W, Wagenaar JA, Owen RJ. Host-pathogen interactions in Campylobacter infections: the host perspective. Clin Microbiol Rev. 2008;21(3):505-18. 
8. Bolton DJ. Campylobacter virulence and survival factors. Food Microbiol. 2015;48:99-108.

9. Galan JE, Waksman G. Protein-injection Machines in Bacteria. Cell. 2018; 172(6):1306-18.

10. Brackmann M, Nazarov S, Wang J, Basler M. Using force to punch holes: mechanics of contractile Nanomachines. Trends Cell Biol. 2017;27(9):623-32.

11. Hachani A, Wood TE, Filloux A. Type VI secretion and anti-host effectors. Curr Opin Microbiol. 2016;29:81-93.

12. Ho BT, Dong TG, Mekalanos JJ. A view to a kill: the bacterial type VI secretion system. Cell Host Microbe. 2014;15(1):9-21.

13. Lertpiriyapong K, Gamazon ER, Feng Y, Park DS, Pang J, Botka G, et al. Campylobacter jejuni type VI secretion system: roles in adaptation to deoxycholic acid, host cell adherence, invasion, and in vivo colonization. PLoS One. 2012;7(8):e42842.

14. Bleumink-Pluym NMC, van Alphen LB, Bouwman LI, Wösten MMSM, van Putten JPM. Identification of a functional type IV secretion system in Campylobacter jejuni conferring capsule polysaccharide sensitive cytotoxicity. PLoS Pathog. 2013;9(5):e1003393.

15. Harrison JW, Dung TT, Siddiqui F, Korbrisate S, Bukhari H, Tra MP, et al. Identification of possible virulence marker from Campylobacter jejuni isolates. Emerg Infect Dis. 2014:20(6):1026-9.

16. De Ceuleneer M, Vanhoucke M, Beyaert R. Role of the bacterial type VI secretion system in the modulation of mammalian host cell immunity. Curr Med Chem 2015;22(14):1734-1744.

17. Filloux A. The rise of the type VI secretion system. F1000Prime Rep. 2013;5:52.

18. Charlson M, Szatrowski TP, Peterson J, Gold J. Validation of a combined comorbidity index. J Clin Epidemiol. 1994;47(11):1245-51.

19. Charlson ME, Pompei P, Ales KL, Mackenzie CR. A new method of classifying prognostic comorbidity in longitudinal studies: development and validation. J Chronic Dis. 1987;40(5):373-83.

20. Radovanovic D, Seifert B, Urban P, Eberli FR, Rickli H, Bertel O, et al. Validity of Charlson Comorbidity Index in patients hospitalised with acute coronary syndrome. Insights from the nationwide AMIS Plus registry 2002-2012. 2014;100(4):288-294.

21. de Groot V, Beckerman H, Lankhorst GJ, Bouter LM. How to measure comorbidity: a critical review of available methods. J Clin Epidemiol. 2003:56(3):221-9.

22. Bessède $E$, Solecki $O$, Sifré E, Labadi L, Mégraud F. Identification of Campylobacter species and related organisms by matrix assisted laser desorption ionization-time of flight (MALDI-TOF) mass spectrometry. 2011; 17(11):1735-39.

23. Corcionivoschi N, Gundogdu O, Moran L, Kelly C, Scates P, Stef L, et al. Virulence characteristics of $h c p(+)$ Campylobacter jejuni and Campylobacter coli isolates from retail chicken. Gut pathogens. 2015;7:20.

24. Ugarte-Ruiz M, Stabler RA, Domínguez L, Porrero MC, Wren BW, Dorell N, et al. Prevalence of type VI secretion system in Spanish Campylobacter jejuni isolates. Zoonoses Public Health. 2015;62:497-500.

25. Gölz G, Rosner B, Hofreuter D, Josenhans C, Kreienbrock L, Löwenstein A, et al. Relevance of Campylobacter to public health--the need for a one health approach. Int J Med Microbiol. 2014;304(7):817-23.

26. Kuhn KG, Nielsen EM, Mølbak K, Ethelberg S. Epidemiology of campylobacteriosis in Denmark 2000-2015. 2018:65(1):59-66.

27. Schmutz C, Mäusezahl D, Jost M, Baumgartner A, Mäusezahl-Feuz M. Inverse trends of Campylobacter and Salmonella in Swiss surveillance data, 19882013. 2016;21(6):30130

28. Nielsen H, Hansen KK, Gradel KO, Kristensen B, Ejlertsen T, Ostergaard C, et al. Bacteraemia as a result of Campylobacter species: a population-based study of epidemiology and clinical risk factors. Clin Microbiol Infect. 2010; 16(1):57-61.

29. Samuel MC, Vugia DJ, Shallow S, Marcus R, Segler S, McGivern T, et al. Epidemiology of sporadic Campylobacter infection in the United States and declining trend in incidence, FoodNet 1996-1999. Clin Infect Dis. 2004; 38(Supplement_3):S165-S74.

30. Sainato R, ElGendy A, Poly F, Kuroiwa J, Guerry P, Riddle MS, et al. Epidemiology of Campylobacter infections among children in Egypt. Am J Trop Med Hyg. 2018;98(2):581-5.

31. Kim J, Lee J, Lee H, Choi JY, Kim DH, Wi YM, et al. Microbiological features and clinical impact of the type VI secretion system (T6SS) in Acinetobacter baumannii isolates causing bacteremia. Virulence. 2017;8(7):1378-89.

32. Luangtongkum T, Jeon B, Han J, Plummer P, Logue CM, Zhang Q. Antibiotic resistance in Campylobacter: emergence, transmission and persistence. Future Microbiol. 2009;4(2):189-200.
33. Collado L, Muñoz N, Porte L, Ochoa S, Varela C, Muñoz I. Genetic diversity and clonal characteristics of ciprofloxacin-resistant Campylobacter jejuni isolated from Chilean patients with gastroenteritis. Genet Evol. 2018;58: 290-3.

34. Kovac J, Cadez N, Stessl B, Stingl K, Gruntar I, Ocepek M, et al. High genetic similarity of ciprofloxacin-resistant Campylobacter jejuni in Central Europe. Front Microbiol. 2015;6:1169.

\section{Ready to submit your research? Choose BMC and benefit from:}

- fast, convenient online submission

- thorough peer review by experienced researchers in your field

- rapid publication on acceptance

- support for research data, including large and complex data types

- gold Open Access which fosters wider collaboration and increased citations

- maximum visibility for your research: over $100 \mathrm{M}$ website views per year

At $\mathrm{BMC}$, research is always in progress.

Learn more biomedcentral.com/submissions 